\section{Efficacy of minimal residual disease driven immune-intervention after allogeneic hematopoietic stem cell transplantation for high-risk chronic lymphocytic leukemia: results of a prospective multicenter trial}

\author{
Olivier Tournilhac, ${ }^{1}$ Magali Le Garff-Tavernier, ${ }^{2}$ Stéphanie Nguyen Quoc, ${ }^{3}$ \\ Edouard Forcade, ${ }^{4}$ Patrice Chevallier, ${ }^{5}$ Faezeh Legrand-Izadifar, ${ }^{6}$ Gandhi \\ Laurent Damaj, ${ }^{7}$ David Michonneau, ${ }^{8}$ Cécile Tomowiak, ${ }^{9}$ Cécile Borel, ${ }^{10}$ \\ Corentin Orvain, ${ }^{11}$ Pascal Turlure, ${ }^{12}$ Rabah Redjou, ${ }^{13}$ Gaëlle Guillerm, ${ }^{14}$ Laure \\ Vincent, ${ }^{15}$ Celestine Simand, ${ }^{16}$ Richard Lemal, ${ }^{17}$ Claire Quiney, ${ }^{2}$ Patricia \\ Combes, ${ }^{18}$ Bruno Pereira, ${ }^{19}$ Laure Calvet, ${ }^{20}$ Aurélie Cabrespine, ${ }^{1}$ Jacques-Olivier \\ Bay, ${ }^{1}$ Véronique Leblond ${ }^{3}$ and Nathalie Dhédin ${ }^{21}$
}

${ }^{1}$ Service d'Hématologie Clinique et de Thérapie Cellulaire, CHU Estaing, Université Clermont Auvergne EA 7453 CIC1405, Clermont-Ferrand; '2Service d'Hématologie Biologique, Groupe Hospitalier Pitié-Salpêtrière, Assistance Publique - Hôpitaux de Paris, Paris; ${ }^{3}$ Service d'Hématologie Clinique, Groupe Hospitalier Pitié-Salpêtrière, Assistance Publique - Hôpitaux de Paris, Paris; ${ }^{4}$ Service d'Hématologie Clinique et de Thérapie cellulaire, $\mathrm{CHU}$ Bordeaux, Bordeaux; ${ }^{5}$ Service d'Hématologie Clinique, $\mathrm{CHU}$ Nantes Hôtel Dieu, Nantes; ${ }^{6}$ Service d'Hématologie Clinique, Département de Greffe de Moelle, CHU Nice, Nice; ${ }^{7}$ Hématologie Clinique, Institut d'Hématologie de BasseNormandie, CHU Côte de Nacre, Caen; ${ }^{8}$ Service Hématologie Greffe, Hôpital Saint-Louis, Assistance Publique - Hôpitaux de Paris, Paris; Université Paris Diderot, Paris; ' $S e r v i c e$ Oncologie Hématologique et Thérapie Cellulaire, CHU Poitiers, Poitiers; ${ }^{10}$ Service d'Hématologie, Institut Universitaire du Cancer Toulouse - Oncopole, Toulouse; ${ }^{11}$ Service Maladies du Sang, CHU Angers, Angers; ${ }^{12}$ Service d'Hématologie Clinique, $\mathrm{CHU}$ Dupuytren, Limoges; ${ }^{13}$ Service d'Hématologie Clinique, Hôpital Henri Mondor, Assistance Publique - Hôpitaux de Paris, Créteil; ${ }^{14}$ Service d'Hématologie Clinique, Institut de Cancéro-Hématologie, Hôpital Augustin Morvan, Brest; ${ }^{15}$ Département Hématologie Clinique, Hôpital St Eloi, Montpellier; ${ }^{16}$ Service Hématologie, CHU de Strasbourg, Strasbourg; ${ }^{17}$ Service d'Histocompatibilité, CHU, Université Clermont Auvergne EA 7453 and CIC501, Clermont-Ferrand; ${ }^{18}$ Service Cytogénétique, CHU Estaing, Clermont-Ferrand; ${ }^{19}$ Unité de Biostatistiques, Direction de la Recherche Clinique (DRCl), CHU, Clermont-Ferrand; ${ }^{20}$ Service de Réanimation Médicale, Hôpital Gabriel Monpied, CHU de Clermont-Ferrand, Clermont-Ferrand and ${ }^{21}$ Unité Adolescents et Jeunes Adultes, Hôpital St Louis, Assistance Publique - Hôpitaux de Paris, Paris, France

\section{ABSTRACT}

A llogeneic hematopoietic stem cell transplantation (HSCT) remains a potentially curative and useful strategy in high-risk relapsing chronic lymphocytic leukemia (CLL). Minimal residual disease (MRD) assessment at 12 months (M12) post-HSCT is predictive of relapse. This phase II study aimed to achieve M12 MRD negativity $\left(\mathrm{MRD}_{\mathrm{n \varepsilon}}\right)$ using an MRD-driven immune-intervention (Md-PII) algorithm based on serial flow-cytometry blood $\mathrm{MRD}$, involving cyclosporine tapering followed in case of failure by donor lymphocytes infusions. Patients had high-risk CLL according to the 2006 European Society for Blood and Marrow Transplantation consensus, in complete or partial response with lymphadenopathy $<5 \mathrm{~cm}$ and comorbidity score $\leq 2$. Donors were HLA-matched sibling or matched unrelated (10/10). Fortytwo enrolled patients with either $17 \mathrm{p}$ deletion (front-line, $\mathrm{n}=11$; relapse $n=16)$ or other high-risk relapse $(n=15)$ received reduced intensity-conditioning regimen before HSCT and were submitted to Md-PII. M12$\mathrm{MRD}_{\mathrm{mg}}$ status was achieved in 27 of 42 patients $(64 \%)$ versus 6 of 42 $(14.2 \%)$ before HSCT. With a median follow-up of 36 months (range, 1953), 3-year overall survival, non-relapse mortality and cumulative incidence of relapse are $86.9 \%$ (95\% Confidence Interval [CI]: 70.8-94.4),
Ferrata Storti Foundation

Haematologica 2021

Volume 106(7):1867-1875

Correspondence:

OLIVIER TOURNILHAC

otournilhac@chu-clermontferrand.fr

Received: October 152019.

Accepted: May 29, 2020.

Pre-published: June 11, 2020.

https://doi.org/10.3324/haematol.2019.239566

(C)2021 Ferrata Storti Foundation

Material published in Haematologica is covered by copyright. All rights are reserved to the Ferrata Storti Foundation. Use of published material is allowed under the following terms and conditions:

https://creativecommons.org/licenses/by-nc/4.0/legalcode. Copies of published material are allowed for personal or internal use. Sharing published material for non-commercial purposes is subject to the following conditions:

https://creativecommons.org/licenses/by-nc/4.0/legalcode, sect. 3. Reproducing and sharing published material for commercial purposes is not allowed without permission in writing from the publisher. 
9.5\% (95\% CI: 3.7-23.4) and 29.6\% (95\% CI: 17.3-47.7). Incidence of 2-year limited and extensive chronic graft versus host disease (cGVHD) is $38 \%$ (95\% CI: $23-53)$ and $23 \%$ (95\% CI: $10-36$ ) including two cases post Md-PII. Fifteen patients converted to $\mathrm{MRD}_{\mathrm{me}}$ either after cyclosporine A withdrawal $(\mathrm{n}=12)$ or after $\mathrm{cGvHD}(\mathrm{n}=3)$. As a time-dependent variable, $\mathrm{MRD}_{\mathrm{nes}}$ achievement at any time-point correlates with reduced relapse (Hazard ratio [HR] 0.14 [range, 0.04-0.53], $P=0.004$ ) and improvement of both progression free (HR 0.18 [range, 0.06-0.6], $P<0.005$ ) and overall (HR 0.18 [range, 0.03-0.98], $P=0.047$ ) survival. These data highlight the value of $\mathrm{MRD}$-driven immune-intervention to induce prompt $\mathrm{MRD}$ clearance in the therapy of CLL (clinicaltrials gov. Identifier: NCT01849939).

\section{Introduction}

Until recently, patients with refractory chronic lymphocytic leukemia (CLL) or who relapse early after purine analogs and rituximab-based chemoimmunotherapy or those harboring 17p deletion (del(17p)) and/or TP53 mutations were considered high-risk patients with reduced overall survival (OS). Better understanding of the molecular and genetic aspects of CLL brought novel and highly active strategies such as targeting kinases downstream of the Bcell receptor (BCR) pathway.1.3 These therapies have profondly modified the CLL therapeutic landscape, thanks to improved efficacy and better tolerability. However, the disease is still incurable and allogeneic hematopoietic stem cell transplantation (HSCT) remains a valid option in selected high-risk patients. ${ }^{4,5}$

Prospective studies have shown that allogeneic HSCT can offer long progression free survival (PFS) and even a cure in $35 \%$ to $45 \%$ of high-risk patients. Reduced intensity conditioning (RIC) HSCT can be proposed to older patients and patients with comorbidities who represent the bulk of the CLL population. However disease recurrence, recorded in $22 \%$ to $46 \%$ patients, is still a major issue. ${ }^{6-9}$ Pre-transplantation refractoriness and bulky disease is associated with higher risk of post-transplantation progression. ${ }^{9,10}$ The level of post-transplantation minimal residual disease (MRD) is widely associated with the risk of further progression. In several studies, a negative $M R D\left(M R D_{m}\right)$ status at 6 to 12 months translated into a progression incidence below $10 \% \cdot{ }^{11-14}$ Moreover, the $\mathrm{MRD}_{\mathrm{ws}}$ status may be reached by post-transplantation immunomodulation such as cyclosporine $\mathrm{A}$ (CsA) tapering or donor lymphocyte infusion (DLI). ${ }^{15}$ These data led us to conduct a prospective study evaluating an approach of RIC HSCT followed by a preemptive MRD-driven immune-intervention with the aim to achieve a $M R D_{\text {sa }}$ status at 12 months post-transplantation.

\section{Methods}

\section{Study design}

The ICLL03 RICAC-PMM (Reduced Intensity Conditioning Allogeneic Transplantation for CLL with Preemptive MDR Management), a joint FILO (French Innovative Leukemia Organization) and SFGM-TC (Société Francophone de Greffe de Moelle et de Thérapie Cellulaire) multicenter phase II trial evaluated the efficacy and safety of a preemptive immune-intervention based on MRD assessment in high-risk CLL. Eligible patients were 18 to 70 years old, with CLL (Matutes score 4 or 5 ) or lymphocytic lymphoma, and high-risk features according to the 2006 European Society for Blood and Marrow Transplantation (EBMT) consensus $^{16}$ (see Online Supplementary Appendix). Patients had to be in complete or partial response with lymphadenopathy $<5 \mathrm{~cm}$ and a comorbidity score $\leq 2$. Donors were HLA-matched sibling or unrelated (10/10). ${ }^{17}$ All responsible Institutional Review Boards in accordance with the Declaration of Helsinki approved the protocol including the study-specific informed consent form. The study was declared to the French Authorities (reference ID-RCB 2011A00906-35) and registered on clinicaltrials gov. Identifier: NCT01849939.

\section{Transplantation modalities}

Conditioning regimen was fludarabine, $30 \mathrm{mg} / \mathrm{m}^{2} /$ day, from day (D) D-5 to D-1, intravenous busulfan $3.2 \mathrm{mg} / \mathrm{kg} /$ day from D4 to D-3 and ATG (thymoglobuline) $2.5 \mathrm{mg} / \mathrm{kg} /$ day from D-3 to D-2. ${ }^{18}$ Stem cell source was G-CSF mobilized peripheral blood cells. Graft-versus-host disease (GvHD) prophylaxis was based on CsA with a short course of methotrexate in case of minor donor/recipient $\mathrm{ABO}$ mismatch.

\section{Response and minimal residual disease evaluation}

Response evaluation was performed according to 2008 iwCLL criteria including computed tomography scan (CT-scan) ${ }^{19}$ before and 3 months (M3), 6 months (M6) and 12 months (M12) after transplantation.

$\mathrm{MRD}$ analysis was centrally performed on blood and/or bone marrow by 10-color multiparameter flow cytometry. ${ }^{20-22} \mathrm{MRD}_{\mathrm{ma}}$ definition was $<1$ CLL cell detectable per 10,000 leukocytes $(<1.10-4) \cdot{ }^{19} \mathrm{MRD}\left(\mathrm{MRD}_{\mathrm{po}}\right)$ definition was $\geq 1 \mathrm{CLL}$ cell detectable per 10,000 leukocytes. Clusters of $<20$ events were considered as undetectable MRD (UD). Blood MRD evaluation was planned before transplantation, then monthly until M6, at M9 and M12. Once achieved, the blood $\mathrm{MRD}_{\mathrm{m} \text { s }}$ status was confirmed 1 month later in both blood and bone marrow.

\section{Preemptive immune-intervention}

Preemptive immune-intervention was applied in the absence of significant GvHD, defined by either acute GvHD (aGVHD) $\geq$ grade 2 or extensive chronic GvHD (cGvHD). The algorithm based on response and blood MRD assessment included acceleration of CsA tapering and withdrawal followed in case of failure by escalating DLI. The algorithm also included extension of CsA treatment in case of early achievement of MRD status (Online Supplementary Appendix; Figure A)

\section{Chimerism and graft-versus-host disease assessment}

Chimerism studies were performed on peripheral blood at M1, M2, M3, M6, and M12 post HSCT by multiplex fluorescent polymerase chain reaction using Short Tandem Repeat analysis. (Online Supplementary Appendix). The diagnosis of GvHD was made according to published criteria. ${ }^{23,24}$

\section{Trial objectives and statistical analysis}

The primary objective was to evaluate the efficacy of a preemptive immune-intervention to achieved $\mathrm{MRD}_{m s}$ at $\mathrm{M} 12$. The probabilities of OS, and PFS were calculated using the KaplanMeier estimator. The probability of non-relapse mortality 
(NRM) and relapse/progression were calculated using the Fine and Gray approach, considering death as competing risks. In order to evaluate the impact on outcomes of $M R D_{m s}$ achievement, we performed time dependent analyses considering $\mathrm{MRD}_{\mathrm{wa}}$ occurrence as a time-dependent event. Outcome data were estimated by the Mantel-Byar method and graphically illustrated by Simon-Makuch plots ${ }^{25,26}$ (Online Supplementary Appendix).

\section{Results}

\section{Patients' characteristics, donor type and protocol adherence}

Between September 2012 and February 2015, 43 patients fulfilling the 2006 EBMT consensus criteria were recruited in 16 French centers; due to donor comorbidities, one patient included was not eventually transplanted. The present analysis includes the remaining 42 patients ( 32 male and 10 female). Patients' characteristics are depicted in the Table 1. Before HSCT, patients had received a median of two

\section{Table 1. Patients' characteristics}

\begin{tabular}{|c|c|}
\hline & Patients ( $n=42)$ \\
\hline $\begin{array}{l}\text { Sex } \\
\quad \text { Female } \\
\text { Male }\end{array}$ & $\begin{array}{l}10 \\
32\end{array}$ \\
\hline Median age at transplant: y (range) & $58.6(40.6-68.6)$ \\
\hline Median time between diagnostic and HSCT: y (range) & $4.5(0.2-14.7)$ \\
\hline $\begin{array}{l}\text { Indication for HSCT: } \mathrm{n} \\
\text { del(17)p and/or TP53 mutation, } 1^{\text {st line }} \\
\text { del(17)p and/or TP53 mutation, in relapse } \\
\text { Purine analogs refractoriness without TP53 abnormality } \\
\text { Early relapse }(<2 \text { y) after fludarabine based combination or } \\
\text { autologous transplant without TP53 abnormality }\end{array}$ & $\begin{array}{l}11 \\
16 \\
3 \\
12\end{array}$ \\
\hline Median prior treatment lines: $\mathrm{n}$ (range) & $2(1-5)$ \\
\hline $\begin{array}{l}\text { Last line before HSCT: } n \\
\text { Alemtuzumab (+/- Dexamethasone) } \\
\text { Bendamustine based combination (B, BR, BOMP) } \\
\text { R-DHAC } \\
\text { Ibrutinib } \\
\text { Idelalisib + rituximab } \\
\text { Rituximab }\end{array}$ & $\begin{array}{l}17 \\
14 \\
6 \\
3 \\
1 \\
1\end{array}$ \\
\hline Median time between last line and HSCT: d (range) & $63(7-179)$ \\
\hline $\begin{array}{l}\text { Prior exposure to alemtuzumab: } \mathrm{n} \\
\text { Median interval between alemtuzumab (last line) and } \\
\text { HSCT: } \mathrm{d} \text { (range) } \\
\text { HSCT done } \leq 60 \text { days post alemtuzumab: } \mathrm{n}\end{array}$ & $\begin{array}{c}20 \\
85(37-179) \\
6\end{array}$ \\
\hline $\begin{array}{l}\text { Disease status at transplantation: } \mathrm{n} \\
\text { CR/CRi } \\
\text { PR } \\
\text { Lymph node }>15 \mathrm{~mm}^{*} \\
\text { Lymph node } \leq 15 \mathrm{~mm}\end{array}$ & $\begin{array}{l}8 \\
34 \\
25 \\
17\end{array}$ \\
\hline $\begin{array}{l}\text { Blood MRD at transplantation } \\
\text { Median MRD level: \% (range) } \\
\text { Negative MRD: } n\end{array}$ & $\begin{array}{c}0.78\left(<10^{4}-70\right) \\
6\end{array}$ \\
\hline $\begin{array}{l}\text { Donor type (HLA 10/10): } n \\
\text { HLA Matched sibling } \\
\text { HLA Matched unrelated } \\
\end{array}$ & $\begin{array}{l}16 \\
26\end{array}$ \\
\hline
\end{tabular}

HSCT: hematopoietic stem cell transplantation; B: bendamustine; BR: bendamustine, rituximab; BOMP: bendamustine, ofatumumab, méthylprednisolone; R-DHAC: rituximab, carboplatin, cytarabine, dexamethasone; CR: complete remission; PR: partial remission; MRD: minimal residual disease. n: number, d: days, m: months, y: years. * No patient with lymph node $>50 \mathrm{~mm}$. lines of treatment (range, 1-5); the last one being alemtuzumab for 17 patients, immunochemotherapy for 21 and BCR inhibitors for four. Details of previous lines of treatment per patients are reported in the Online Supplementary Table S1. Eight patients were in CR/CRi (including six with blood $\mathrm{MRD}_{w}$ status) and 34 in PR pre-transplantation. Donors were HLA-identical siblings $(\mathrm{n}=16)$ or HLAmatched $(10 / 10)$ unrelated donors $(n=26)$.

The trial profile of the immune-intervention applied in this study and the representative protocol adherence is shown in the Online Supplementary Appendix (Online Supplementary Figure 1B). One patient died before D30. Among the 41 remaining patients, seven were not treated strictly according to study protocol: four patients had an unplanned early CsA withdrawal for primary $(n=1)$ or secondary $(n=1)$ graft failure or mixed chimerism $(n=2)$. One of these last two patients relapsed at 13 months, whereas the second, who later received DLI, was still in mixed chimerism without relapse at 18 months. For two patients, CsA was tapered early, despite $\mathrm{MRD}_{\mathrm{ws}}$ status, due to renal failure in one patient. Finally CsA was reduced at D120 instead of D90 for one patient with $\mathrm{D} 90 \mathrm{MRD}_{\mathrm{rax}}$ status.

\section{Post-transplantation response and outcomes}

Engraftment occurred in 40 of the 42 transplanted patients while two presented graft failure. In the latter patients, both in PR at transplantation, the last line were alemtuzumab plus dexamatasone, interrupted 49 days before transplant in one patient and ibrutinib interrupted 9 days before transplant in the other. Assessment of the response according to the iwCLL criteria between 3 and 6 months found CR/CRi and PR for $13(31 \%)$ and $26(62 \%)$ patients, respectively. Response was not evaluated for three patients because of early death $(n=1)$ or graft rejection $(\mathrm{n}=2)$. Response was classified as partial when there was lymph node $>15 \mathrm{~mm}$ persistence $(\mathrm{n}=6)$, spleen enlargement $(n=4)$, both $(n=3)$ or incomplete evaluation $(n=13)$ (Online Supplementary Table S2).

Seventeen patients developed grade $1(\mathrm{n}=8), 2(\mathrm{n}=6)$ and $3(\mathrm{n}=3) \mathrm{aGvHD}$. Limited and extensive cGvHD occurred in 15 and nine of the 39 patients who engrafted and were still alive at D100, translating into a cumulative incidence at 2 years of cGvHD of $61 \%$ (95\% Confidence Interval [CI]: 54 68), including limited and extensive cGvHD in 38\% (95\% CI: $23-53$ ) and in $23 \%$ (95\% CI: $10 \%-36 \%$ ) patients respectively. Two cases of primary cGvHD were diagnosed following planned immune-intervention: one was a limited cGVHD after DLI administration for early progression at D35 and one was an extensive cGvHD after cessation of CsA due to D90 MRD positivity.

Seven of the 42 patients died. Causes of death were extensive cGvHD $(\mathrm{n}=2)$, pulmonary aspergillosis plus Pneumocystis jiroveci pneumonia associated with limited $\mathrm{cGvHD}(\mathrm{n}=1)$ and early cytomegalovirus infection $(\mathrm{n}=1)$ in a patient who received alemtuzumab in the last weeks prior to transplantation. The three remaining deaths were related to disease progression with Richter transformation. Moreover three patients presented severe complications, namely two polyradiculopathy and one Epstein-Barr virusinduced lymphoproliferative disease.

With a median follow-up of survivors of 36 months (range, 19-53) the 3-year OS, PFS, and NRM were 86.9\% (95\% CI: 70.8-94.4), $62.9 \%$ (95\% CI: $45.8-75.9)$ and $9.5 \%$ (95\% CI: 3.7-23.4) respectively. Ten patients had progression occurring after a median of 12 months (range, 1-34). 
The 3-year cumulative incidence of relapse was 29.6\% (95\% CI: 17.3-47.7) (Figure 1). Salvage therapy was delivered in patients who relapsed after donor engraftment; eight patients received ibrutinib; six of them are still in remission at the last follow-up (32 to 52 months) while two had a transient response followed by progression with Richter transformation. (Table 2).

\section{Minimal residual disease (MRD) status at 12 months and MRD kinetics after hematopoietic stem cell transplantation}

At $\mathrm{M} 12,27$ patients achieved $\mathrm{MRD}_{\text {neg }}$ status, including 23 patients with an undetectable MRD $(M R D<$ limit of detection), seven patients remained $M R D_{\text {pos }}$, eight patients were not evaluable because either early toxic death $(n=4)$ or other reason including graft rejection $(n=2)$, EppsteinBarr virus-induced lymproliferation $(n=1)$ and early relapse $(n=1)$. Thus, at $M 12, M^{2} D_{\text {neg }}$ status was achieved in $64 \%$ (27 of 42 ) if we consider all patients and in $77 \%$ (27of 35) if we take into consideration all 34 patients assessed at this time point and the patient who experienced a clinical relapse at 1 month (and thus not subject to systematic MRD assessment but considered as failure) versus $14.2 \%$ before transplantation. Most patients remained $\mathrm{MRD}_{\text {pos }}$ early after transplantation and progressively translated to $\mathrm{MRD}_{\text {neg }}$ within the first 6 months posttransplantation. (Figure 2). Nine of the 13 (69\%) D90
$\mathrm{MRD}_{\text {pos }}$ patients who had no significant GvHD but who had an early CsA withdrawal according to the protocol, managed to reach a $\mathrm{MRD}_{\text {neg }}$ status.

For the 39 patients who engrafted and were alive after M1, MRD kinetics followed four distinctive patterns. (Figure 3$)$. The pattern $A(n=6)$ is constituted of the pretransplantation $\mathrm{MRD}_{\text {nes }}$ patients. Two of these patients relapsed, one at 12 and one at 19 months. The pattern $B$ $(n=11)$ comprised the patients who converted from pretransplantation $\mathrm{MRD}_{\text {pos }}$ to post-transplantation $\mathrm{MRD}_{\text {ne }}$ status within 3 months without any immune-intervention. One pattern-B patient with M12 MRD close to the positivity threshold relapsed at 13 months. The pattern $C(n=15)$ is constituted of the patients with pre-transplantation $M R D_{\text {pos }}$ who remained $M R D_{\text {pos }}$ during the first 3 months but became $\mathrm{MRD}_{\text {neg }}$ either after CsA tapering and withdrawal $(n=12)$ or after $\mathrm{cGvHD}(\mathrm{n}=3)$. Two pattern- $C$ patients relapsed at 23 and 34 months. The pattern $\mathrm{D}(\mathrm{n}=7)$ comprised the patients with a pre-transplantation $\mathrm{MRD}_{\text {pos }}$ status who remained $M R D_{\text {pos }}$ despite cGvHD $(n=1)$ or immune-intervention including CsA tapering and withdrawal $(n=6)$ followed by DLI for five of them. Progression was observed in five pattern-D patients including three Richter transformations, each occurring within the first 13 months. The outcome of all four panels is represented in the Online Supplementary Table S3.
A

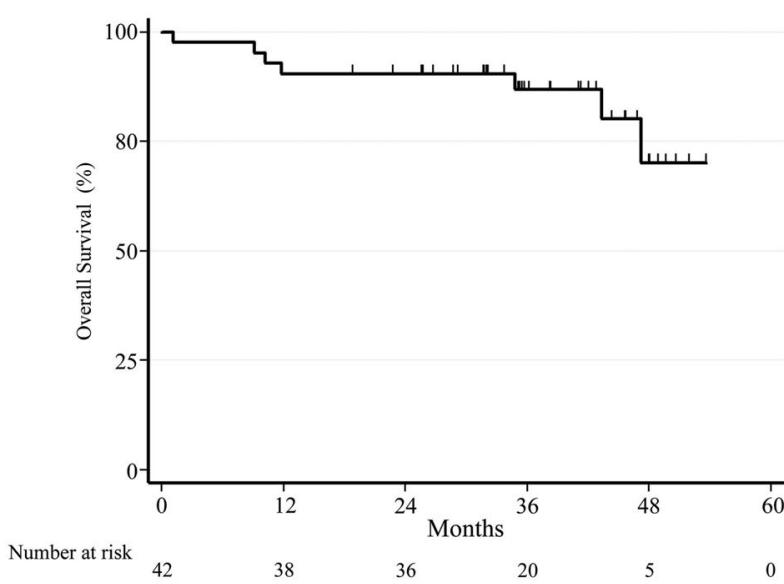

B

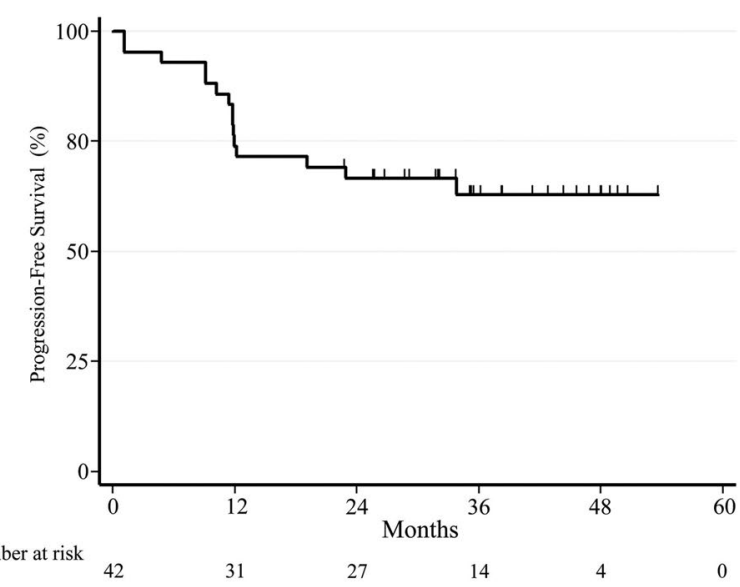

C

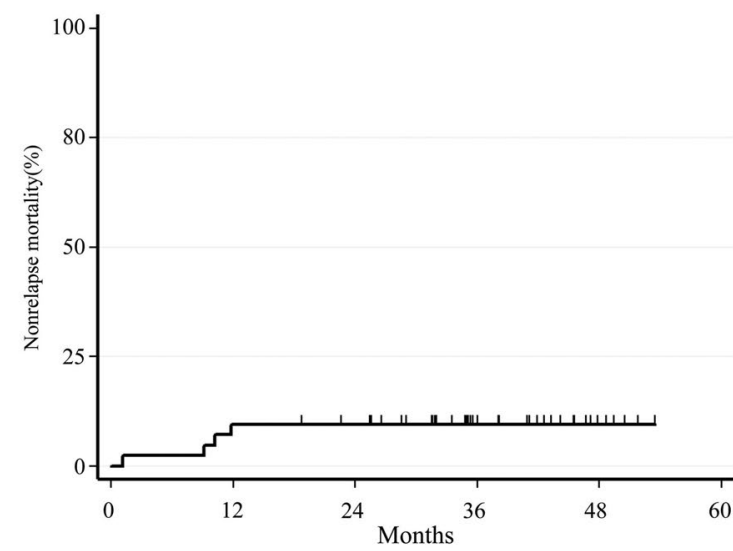

D

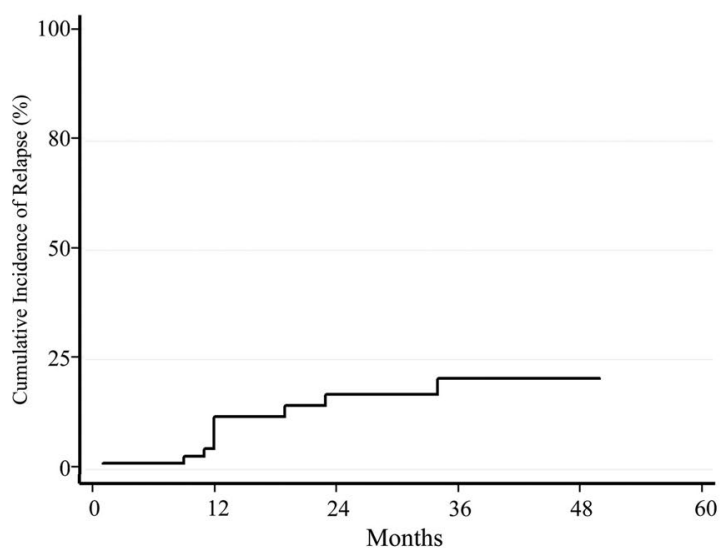

Figure 1. Post-transplant outcome of the 42 chronic lymphocytic leukemia transplanted patients. Kaplan-Meier estimates of (A) overall survival, (B) progressionfree survival. Calculated probability of (C) non relapse mortality and (D) cumulative incidence of relapse after hematopoietic stem cell transplantation. 


\section{Impact of minimal residual disease on outcome}

In Mantel-Byar analysis, evaluating $\mathrm{MRD}$ as a timedependent variable, achievement of the $\mathrm{MRD}_{w_{i}}$ status regardless of the time point, was predictive of an improved PFS, Hazard ratio (HR) 0.18 (range, 0.06-0.60), $P=0.005$, and OS, HR 0.18 (range, 0.03-0.98), $P=0.047$ along with a reduction of CIR, HR 0.14 (range, 0.040.539, $P=0.004$. (Figure 4).

\section{Chimerism evaluation}

The chimerism analyzed on unselected blood cells had no impact on outcome. Conversely T-cell donor engraftment $(\geq 95 \%$ donor $T$ cells) tended to be associated with higher PFS, HR 0.16 (range, 0.02-1.37), $P=0.09$, and lower relapse risk, HR 0.16 (range, $0.02-1.22$ ) $P=0.08$ but had no impact on OS, $P=0.18$.

\section{Discussion}

Since the first descriptions, allogeneic HSCT has long been the only curative treatment for CLL. Its development has benefited greatly from the advent of reduced-intensity conditioning that can be proposed until the age of 70 . The availability of new alternative therapies, including both BCR and BCL-2 inhibitors have in high-risk patients taken the place of allogeneic HSCT and delayed this strategy until later in the management of the disease. Consequently, the

Table 2. Treatment and follow-up of patients in relapse after hematopoietic stem cell transplantation.

\begin{tabular}{|c|c|c|c|c|c|}
\hline Pt\# & $\mathrm{T} 1$ & 12 & Relapse treatment & Last FU & Status \\
\hline 35 & 12 & 13 & Ibrutinib (M13-ongoing) & $29+$ & $\mathrm{PR}, \mathrm{MRD}_{\mathrm{pa}}$ \\
\hline 2 & 19 & 34 & DLI(1), failure followed by ibrutinib (M36-ongoing) & $52+$ & $\mathrm{CR}, \mathrm{MRD}_{\mathrm{ma}}$ \\
\hline 8 & 12 & 15 & DLI(3) followed by Ibrutinib (M18 - ongoing) & $48+$ & $\mathrm{CR}, \mathrm{MRD}_{\text {mas }}$ \\
\hline 19 & 34 & 34 & Ibrutinib (M34 - ongoing) & $42+$ & $\mathrm{CR}, \mathrm{MRD}_{\text {mas }}$ \\
\hline 29 & 23 & 23 & DLI(2) response, followed by ruxolitinib (ongoing) for GvHD & $36+$ & $\mathrm{CR}, \mathrm{MRD}_{\text {mas }}$ \\
\hline 13 & $11^{*}$ & 19 & RCHOP, with initial PR, RDHAP, irradiation & 35 & $\mathrm{PD}^{*}$ \\
\hline 4 & 1 & 6 & ${ }^{\circ}$ Ibrutinib (M15-M45) with initial PR then PD*, RDHAC & 47 & PD* \\
\hline 15 & 12 & 12 & ${ }^{\circ}$ Ibrutinib (M13-M24) with initial PR then PD*, RCHOP & 43 & $\mathrm{PD}^{*}$ \\
\hline 28 & 12 & 13 & Ibrutinib (M13-ongoing) & $32+$ & $\mathrm{CR}, \mathrm{MRD}_{\text {mas }}$ \\
\hline 18 & 9 & 10 & Ibrutinib (M10-ongoing) & $41+$ & $P R, M D_{m a}$ \\
\hline
\end{tabular}

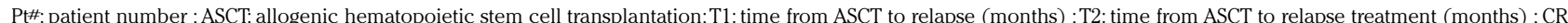
complete response ;PR: partial response ;PD:progressive disease ; *: Richter transformation ;DLI: donor lymphocytes infusion (number) ; R-CHOP: rituximab, cyclophosphamide, doxorubicin, vincristine, prednisone ; R-DHAC: rituximab, dexamethasone, cytarabine, carboplatinum; GvHD: graft versus host disease; FU: follow-up; M13: 13 months; M15: 15 months; M24: 24 months; M34:34 months; M45: 45 months., ${ }^{\circ}$ Patients $\# 4$ and \#15 had received preemptive DLI before relapse treatment as part of the immune-intervention as per study protocol

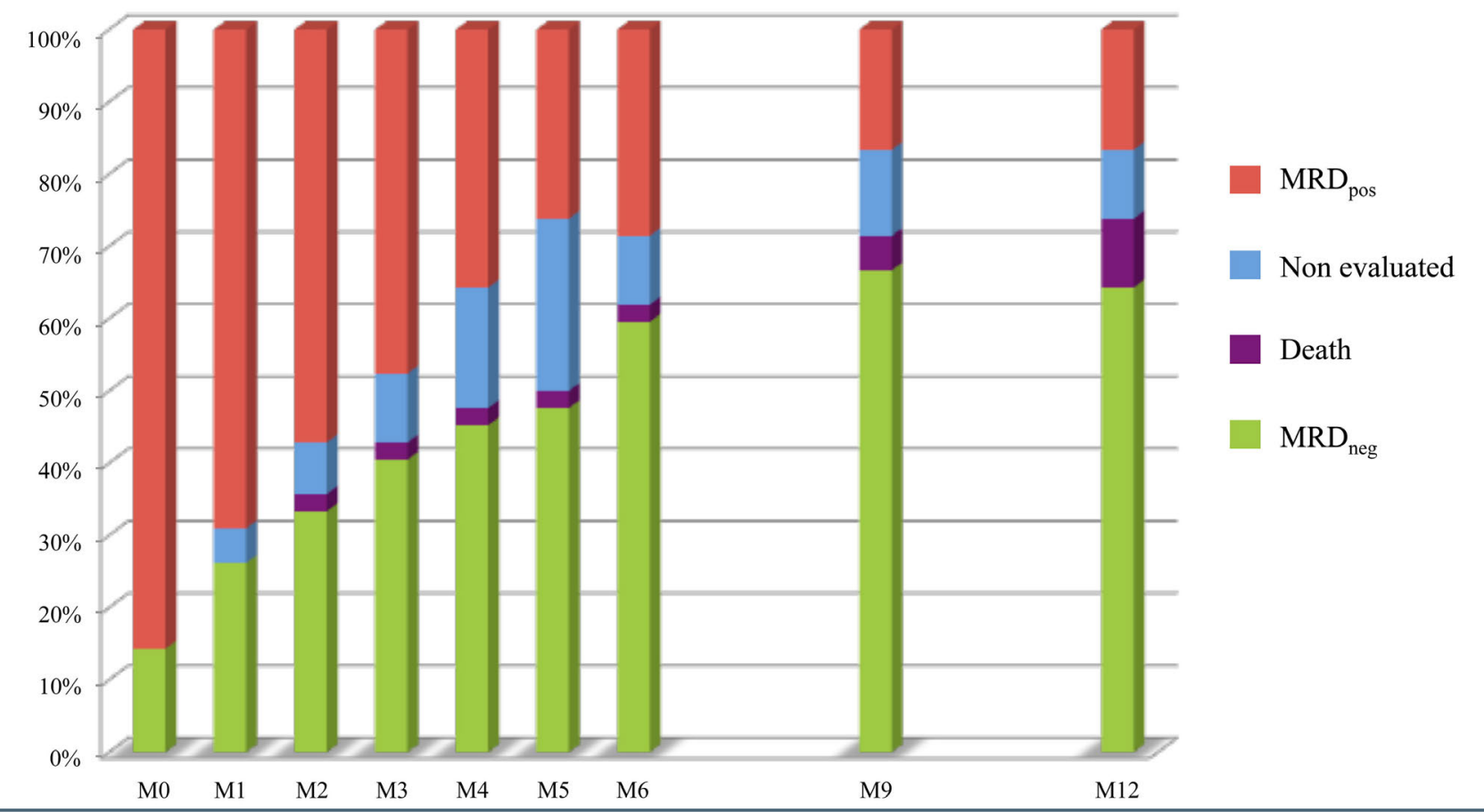

Figure 2. Post-transplantation minimal residual disease evaluation. At 12 months (M12), 27 of 42 (64\%) patients were minimal residual disease negative (MRD neg), 7 of $42(17 \%)$ patients remained MRD positive (MRD $\left.{ }^{\circ}\right), 8$ of $42(19 \%)$ patients were not evaluable because either prior early toxic death ( $\left.\mathrm{n}=4\right)$ and 4 of 42 patients $(9.5 \%)$ or other reasons including graft rejection $(n=2)$, Eppstein-Barr virus lymproliferation $(n=1)$ and early relapse $(n=1)$ 
A

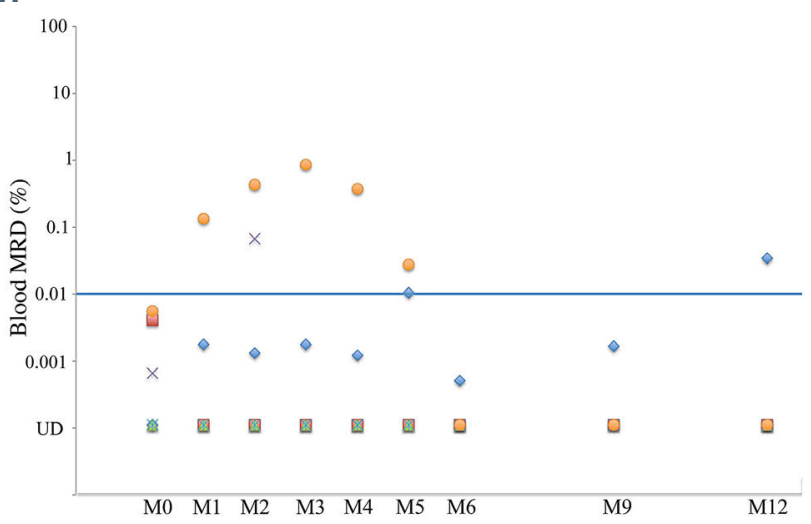

C

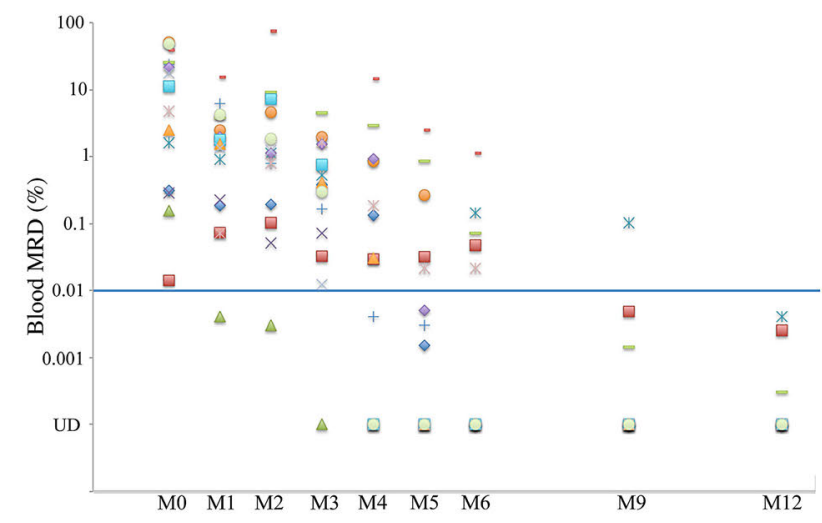

B

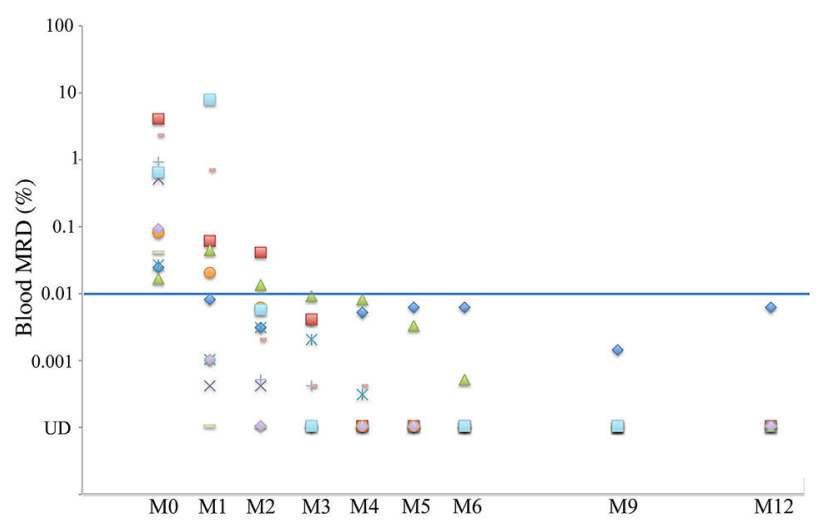

D

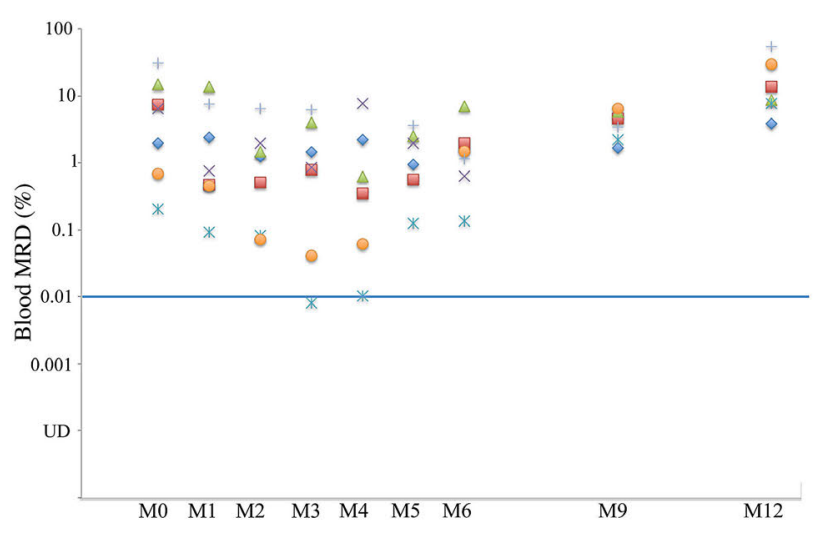

Figure 3. Patterns of minimal residual disease response of the 39 patients who engrafted and were alive after 1 month. Pattern A: patients with pre-transplant minimal residual disease negative (MRD $\left.{ }^{\text {neg }}\right)$ status $(n=6)$, Pattern B: patients who converted to MRD $^{\text {neg }}$ within 3 months post-transplant without any immune-intervention $(n=11)$. Pattern $C$ : patients who converted to $M_{R D}$ upon immune-intervention (cyclosporine A [CsA] withdrawal only) or graft-versus-host disease $(G v H D)$ ( $\left.n=16\right)$ Pattern D: patients who remained MRD positive (MRD $D_{\text {ses }}$ ) during follow-up despite immune-intervention (CsA withdrawal and donor lymphocyte infusion [DLI]) or GvHD $(n=7)$. Solid blue line: negativity limit of MRD $(<0.01 \%)$. UD : undetectable MRD (MRD < limit of detection [LOD]).

number of allogeneic HSCT for CLL has considerably decreased since 2015, both in the United States ${ }^{27}$ and Europe. $^{28}$ BCR and BCL-2 inhibitors allow control of relapsed CLL with a response duration exceeding those reported after immunochemotherapy. ${ }^{29,30}$ However, relapses are the rule, particularly in patients with adverse molecu$\operatorname{lar}^{31,32}$ and/or complex karyotype. ${ }^{33,34}$ For such patients, CAR-T cells are also a hope. ${ }^{35,36}$ However, while this new option is very promising in several hematological diseases, in the 134 highly pre-treated CLL reported to date, the complete response rate remains 20 to $30 \%$, with a median PFS of $18 \%$ at 18 months. ${ }^{37}$ This approach is associated with significant acute toxicity, but does not present, in contrast to allogeneic HSCT, a risk of GvHD. Hence, long-term results in large cohorts of CLL patients treated by CAR-T cells are currently needed, and allogeneic HSCT is still a valid option in CLL for selected patients. ${ }^{38}$

We report the first trial evaluating prospectively an approach of post-transplantation MRD-driven immuneintervention for CLL. M12 MRD $\mathrm{mos}_{\xi}$ associated with a reduced risk of relapse and an improvement of disease-free survival, was chosen as the primary endpoint. ${ }^{10-14} \mathrm{We}$ hypothesized that early CsA tapering potentially followed

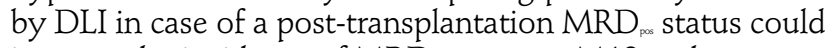
increase the incidence of $\mathrm{MRD}_{\mathrm{mg}}$ status at $\mathrm{M} 12$ and as a consequence could reduce the risk of relapse. Conversely, for patients with a post-transplantation $\mathrm{MRD}_{\mathrm{ws}}$ status, CsA administration would be extended for a longer period to reduce the risk of chronic GvHD.

In order to minimize severe GvHD incidence, we selected the same ATG containing conditioning regimen as previously evaluated in a large multicenter study performed in a similar age population..$^{18}$ Overall, in this population with a median age close to 60 years, we observed less than $25 \%$ extensive $\mathrm{cGvHD}$ which appears lower than in previous series of HSCT in CLL and can be considered very acceptable in the context of allogeneic treatment of high-risk diseases. ${ }^{7 \cdot 10}$

Moreover, only four deaths were related to either GvHD or infection and the 2-year NRM less than 10\%, favorably compares with those varying from $17 \%$ to $27 \%$ reported in the main series of reduced intensity conditioning transplant in CLL. ${ }^{7-10}$ In this trial low NRM highly contribute to impressive 3-year OS close to $90 \%$.

We show that post-transplantation MRD-driven immune-intervention is feasible in the setting of a multicenter trial. MRD evaluation was centralized and performed by a sensitive method of high-resolution ten-color flow cytometry. Results were available within 48 hours, allowing a rapid adaptation of the immunosuppressive therapy. Immune-intervention was conducted in accordance to the protocol in $83 \%$ of the evaluable patients; in the remaining patients CsA was withdrawn earlier due to graft-failure, 
A

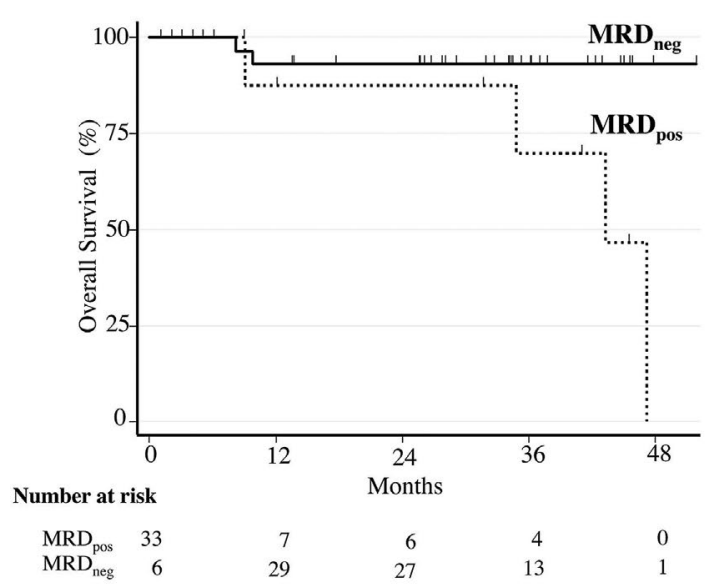

C

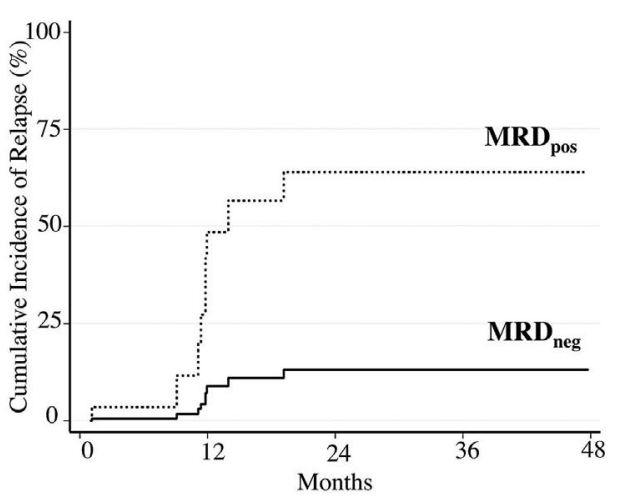

mixed chimerism or renal failure. The immune-intervention related toxicity was low, with only two cases of GvHD occurring after CsA withdrawal or DLI applied as per protocol.

In an Intent-To-Treat analysis, the primary end-point of M12 $\mathrm{MRD}_{\mathrm{wa}}$ status has been achieved in $64 \%$ of the 42 transplanted patients and in $79 \%$ of the 34 patients who actually had a M12 MRD evaluation. This result favorably compares with both prospective $e^{10}$ and retrospective $e^{11-14}$ studies reporting $48 \%$ to $71 \% \mathrm{MRD}_{\text {ws }}$ status at 6 to 12 months after HSCT..$^{10-14}$ Particularely our results are in line with one large single-center retrospective analysis of 77 allografted CLL patients submitted to immune modulation based on MRD evaluation. ${ }^{6}$ In this latter study M12 MRD clearance was achieved in $56 \%$ overall and $84 \%$ of all patients evaluable for M12 MRD status and the 3-year relapse incidence was $26 \%$. Our data argue for the benefit of an early preemptive immune-intervention based on MRD evaluation. Thus, early CsA withdrawal applied in D90 MRD ${ }_{\text {re }}$ patients translated into $\mathrm{MRD}_{\mathrm{ws}}$ status at $\mathrm{M} 12$ in $69 \%$ of them. Moreover, most patients with D90 MRD ${ }_{\text {pex }}$ status and GvHD, spontaneously switched to $\mathrm{MRD}_{\mathrm{wa}}$ at $\mathrm{M} 12$, highlighting the role of allogeneic reaction in the control of the disease. Conversely, in this context of early preemptive immune-intervention we failed to show a benefit of DLI to convert MRD from positive to negative, but three of five patients were already in clinical progression at the time of infusion. Finally, considering the non-randomized nature of
B

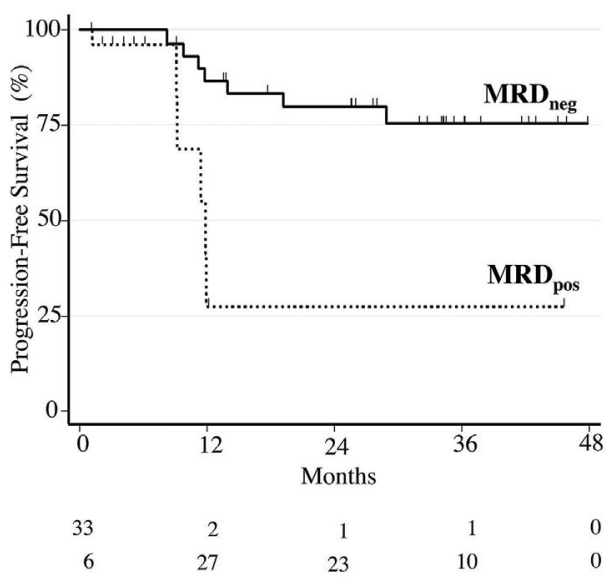

Figure 4. Impact of minimal residual disease negative $\left(M_{R} D_{m s}\right)$ status achievement on post transplant outcome according to the Mantel-Byar method illustrated by Simon-Makuch plots (MRD status as a time-dependent event). (A) overall survival, (B) progression-free survival and (C) cumulative incidence of relapse.

the trial, our data suggest that a MRD-driven CsA withdrawal can provide durable MRD clearance, improve GvHD leukemia effect and avoid progression.

In several studies, the M12 MRD ${ }_{m \text { so }}$ status was associated with lower incidence of relapse..$^{13-15,39}$ As half of the progression occurred before M12 in our series, we chose to analyze the impact of $M R D$ using $M R D$ as a time dependent variable. We confirm here the strong correlation between an $\mathrm{MRD}_{\mathrm{ma}}$ status achievement regardless of time point and both low progression and better PFS. Interestingly, we also show that $\mathrm{MRD}_{\mathrm{mg}}$ status achievement translates into better survival. The impressive post-transplant OS closed to $90 \%$ at 3 years in this high-risk CLL population could also be explained by the possibility opened to physician of treating post-allograft relapses with ibrutinib. It should also be noted that the three patients who died from CLL-related cause had Richter's syndrome, including two escaping therapy with ibrutinib.

These data lead us to propose early additional therapy in patients who display an $\mathrm{MRD}_{\text {w }}$ status despite either MRDdriven CsA withdrawal or chronic GvHD, or in the rare patients who could experience disease despite $\mathrm{MRD}_{\mathrm{wz}}$ status achievement. Recent reports show the efficacy of ibrutinib in post-HSCT CLL relapse without limiting toxicity or GvHD, as also observed in our series. ${ }^{40,41}$ This treatment should be evaluated preemptively in patients who fail to achieve negative MRD after CsA cessation.

In conclusion, this report shows the feasibility of MRD- 
driven immune-intervention following ATG-based RIC allogeneic HSCT in CLL. These data highlight the importance to repeatedly monitor post-HSCT MRD to guide early CsA discontinuation in patients with $\mathrm{D} 90 \mathrm{MRD}_{\text {pe }}$ and without GvHD. However, as we report the results of this study in 2020, we must emphasize that the entry criteria were based on the EBMT 2007 recommendations, which no longer represent current practice. Particularly, this is the case for untreated patients with del(17p) and/or TP53 mutation who represent $26 \%$ of the study's enrollment. A presentation of post-transplant outcome excluding these 11 patients is shown in the Online Supplementary Appendix (Online Supplementary Figure S3). Allogeneic HSCT indications have evolved in 2014 under the impulse of the European Research Initiative on CLL (ERIC) and EBMT with of a new decisional algorithm according to patient biology and prior treatment with BCR and BCL-2 inhibitors, ${ }^{42}$ the feasibility of which has just been reported in a recent analysis. ${ }^{43}$ The pre-emptive immune modulation based on post-transplant MRD, as described in our study in patients who were $90 \%$ naive of BCR and/or BCL-2 treaments, should also be effective in patients pretreated with such agents, but this will have to be demonstrated.

\section{Disclosures}

OT has received travel grant, scientific support, and honorarium for board participation from Amgen, Roche, Jansen, Abbevie and Gilehead; MLGT has receieved honorarium for board participation from Alexion; VL has received board participation, speaker bureau, honorarium from Roche, Abbevie, Gilehead, Amgen and Janssen.

\section{Contributions}

$O T$ and ND designed and performed research including patients' care, coordinated the study and wrote the paper; MLGT and CQ performed MRD flow cytometry assessment; $P C$ performed molecular chimerism; SNGQ, EF, PC, FLI, GLD, DM, $C T, C B, C O, P T, R R, G G, L V, C S, J O B, V L$ performed research especially patients' care; $R L, L C$, and $A C$ worked on study design, study application and national coordination and BP performed statistical data analysis.

\section{Acknowledgments}

The study was supported by a National Grant from the Fondation ARC. Logistic support was provided through the Fonds de recherche clinique en hématologie Force Hémato. Les Laboratoires Pierre Fabre provided financial research support for the study, but did not participate in the conduct of the study or data and results analysis.

The authors thank Dr Reza Tabrizi and Dr Oumeladi Reman for their devoted implication in patient care and the clinical research assistants of the FILO group for the onsite monitoring process performed during the study.

\section{References}

1. Byrd JC, Furman RR, Coutre SE, et al. Targeting BTK with ibrutinib in relapsed chronic lymphocytic leukemia. N Engl J Med. 2013;369(1):32-42.

2. Furman RR, Sharman JP, Coutre SE, et al. Idelalisib and rituximab in relapsed chronic lymphocytic leukemia. N Engl J Med. 2014:370(11):997-1007.

3. Roberts AW, Davids MS, Pagel JM. et al. Targeting BCL2 with venetoclax in relapsed chronic lymphocytic leukemia. N Engl J Med. 2016;374(4):311-322.

4. Gribben JG. How and when I do allogeneic transplant in CLL. Blood 2018; 132(1):31-39.

5. Hallek M. Chronic lymphocytic leukemia: 2017 update on diagnosis, risk stratification, and treatment. Am J Hematol. 2017;92(9):946-965.

6. Hahn M, Böttcher S, Dietrich S, et al. Allogeneic hematopoietic stem cell transplantation for poor-risk CLL: dissecting immune-modulating strategies for disease eradication and treatment of relapse. Bone Marrow Transplant. 2015;50(10):1279-1285.

7. Khouri IF, Bassett R, Poindexter N, et al. Nonmyeloablative allogeneic stem cell transplantation in relapsed/refractory chronic lymphocytic leukemia. Cancer. 2011;117(20):4679-4688.

8. Michallet M, Socié G, Mohty M, et al. Rituximab, fludarabine, and total body irradiation as conditioning regimen before allogeneic hematopoietic stem cell transplantation for advanced chronic lymphocytic leukemia: long-term prospective multicenter study. Exp Hematol. 2013;41(2):127-133.

9. Sorror ML, Storer BE, Sandmaier BM, et al. Five-year follow-up of patients with advanced chroniclLymphocytic leukemia treated with allogeneic hematopoietic cell transplantation after nonmyeloablative con- ditioning. J Clin Oncol. 2008;26(30):49124920.

10. Dreger P, Döhner $H$, Ritgen $M$, et al. Allogeneic stem cell transplantation provides durable disease control in poor-risk chronic lymphocytic leukemia: long-term clinical and MRD results of the German CLL Study Group CLL3X trial. Blood. 2010;116(14):2438-2447.

11. Algrin C, Golmard J-L, Michallet M, et al. Flow cytometry minimal residual disease after allogeneic transplant for chronic lymphocytic leukemia. Eur J Haematol. 2017; 98(4):363-370.

12. Farina L, Carniti C, Dodero A, et al. Qualitative and quantitative polymerase chain reaction monitoring of minimal residual disease in relapsed chronic lymphocytic leukemia: early assessment can predict longterm outcome after reduced intensity allogeneic transplantation. Haematologica. 2009;94(5):654-662

13. Logan AC, Zhang B, Narasimhan B, et al. Minimal residual disease quantification using consensus primers and high-throughput IGH sequencing predicts post-transplant relapse in chronic lymphocytic leukemia. Leukemia. 2013;27(8):1659-1665.

14. Moreno C, Villamor N, Colomer D, et al. Clinical significance of minimal residual disease, as assessed by different techniques, after stem cell transplantation for chronic lymphocytic leukemia. Blood. 2006;107(11):4563-4569.

15. Ritgen M, Böttcher S, Stilgenbauer S, et al. Quantitative MRD monitoring identifies distinct GVL response patterns after allogeneic stem cell transplantation for chronic lymphocytic leukemia: results from the GCLLSG CLL3X trial. Leukemia. 2008;22(7):1377-1386.

16. Dreger P, Corradini P, Kimby E, et al. Indications for allogeneic stem cell trans- plantation in chronic lymphocytic leukemia: the EBMT transplant consensus. Leukemia. 2007;21(1):12-17.

17. Sorror ML, Maris MB, Storb R, et al. Hematopoietic cell transplantation (HSCT) specific comorbidity index: a new tool for risk assessment before allogeneic HSCT. Blood. 2005;106(8):2912-2919.

18. Blaise D, Devillier R, Lecoroller-Sorriano AG, et al. Low non-relapse mortality and long-term preserved quality of life in older patients undergoing matched related donor allogeneic stem cell transplantation: a prospective multicenter phase II trial. Haematologica. 2015;100(2):269-274.

19. Hallek M, Cheson BD, Catovsky D, et al. Guidelines for the diagnosis and treatment of chronic lymphocytic leukemia: a report from the International Workshop on Chronic Lymphocytic Leukemia updating the National Cancer Institute-Working Group 1996 guidelines. Blood. 2008;111(12):5446-5456.

20. Rawstron AC, Villamor N, Ritgen M, et al. International standardized approach for flow cytometric residual disease monitoring in chronic lymphocytic leukaemia. Leukemia. 2007;21(5):956-964.

21. Rawstron AC, Böttcher S, Letestu R, et al. Improving efficiency and sensitivity: European Research Initiative in CLL (ERIC) update on the international harmonised approach for flow cytometric residual disease monitoring in CLL. Leukemia. 2013;27(1):142-149.

22. Rawstron AC, Fazi C, Agathangelidis A, et al. A complementary role of multiparameter flow cytometry and high-throughput sequencing for minimal residual disease detection in chronic lymphocytic leukemia: an European Research Initiative on CLI study. Leukemia. 2016;30(4):929-936.

23. Glucksberg H, Storb R, Fefer A, et al. Clinical 
manifestations of graft-versus-host disease in human recipients of marrow from HL-Amatched sibling donors. Transplantation. 1974;18(4):295-304.

24. Przepiorka D, Weisdorf D, Martin P, et al. 1994 consensus conference on acute GVHD grading. Bone Marrow Transplant. 1995;15 (6):825-828.

25. Mantel N, Byar DP. Evaluation of responsetime data involving transient states: an illustration using heart-transplant data. J Am Stat Assoc. 1974;69:81-86.

26. Simon R, Makuch RW. A non-parametric graphical representation of the relationship between survival and the occurrence of an event: application to responder versus nonresponder bias. Stat Med. 1984;3(1):35-44.

27. Kharfan-Dabaja MA, Kumar A, Hamadani $\mathrm{M}$, et al. Clinical practice recommendations for use of allogeneic hematopoietic cell transplantation in chronic lymphocytic leukemia on behalf of the guidelines committee of the American Society for Blood and Marrow Transplantation. Biol Blood Marrow Transplant. 2016;22(12):2117-2125.

28. Tournilhac O, van Gelder N, Dreger P, et al. The 10-years EBMT landscape of allogeneic hematopoietic cell transplantation (alloHCT) for chronic lymphocytic leukemia. 46th Annual Meeting of the European Society for Blood and Marrow Transplantation. Madrid, 2020

29. Chanan-Khan A, Cramer P, Demirkan F, et al. Ibrutinib combined with bendamustine and rituximab compared with placebo, bendamustine, and rituximab for previously treated chronic lymphocytic leukaemia or small lymphocytic lymphoma (HELIOS): a randomised, double-blind, phase 3 study. Lancet Oncol. 2016;17(2):200-211.

30. Deng R, Gibiansky L, Lu T. Bayesian population model of the pharmacokinetics of venetoclax in combination with rituximab in patients with relapsed/refractory chronic lymphocytic leukemia: results from the Phase III MURANO Study. Clin Pharmacokinet. 2019;58(12):1621-1634.

31. O'Brien S, Furman RR, Coutre S, et al. Single-agent ibrutinib in treatment-naïve and relapsed/refractory chronic lymphocytic leukemia: a 5-year experience. Blood. 2018;131(17):1910-1919.

32. Mato AR, Thompson M, Allan JN, et al. Real-world outcomes and management strategies for venetoclax-treated chronic lymphocytic leukemia patients in the United States. Haematologica. 2018;103(9):15111517.

33. Jain P, Thompson PA, Keating M, et al. Longterm outcomes for patients with chronic lymphocytic leukemia who discontinue ibrutinib. Cancer. 2017;123(12):2268-2273.

34. Anderson M, Tam C, Lew TE, et al. Clinicopathological features and outcomes of progression of CLL on the BCL2 inhibitor venetoclax. Blood. 2017;129(25):3362-3370.

35. Porter DL, Hwang W-T, Frey NV, et al. Chimeric antigen receptor $\mathrm{T}$ cells persist and induce sustained remissions in relapsed refractory chronic lymphocytic leukemia. Sci Transl Med. 2015;7(303):303ra139

36. Turtle CJ, Hay KA, Hanafi LA, et al. Durable molecular remissions in chronic lymphocytic Leukemia treated with CD19-specific chimeric antigen receptor-modified $\mathrm{T}$ cells after failure of ibrutinib. J Clin Oncol. 2017;35(26):3010-3020.

37. Lemal R. Tournilhac O. State-of-the-art for CAR T-cell therapy for chronic lymphoid leukemia in 2019. J Immunother Cancer. 2019;7(1):202

38. Dreger P, Ghia P, Schetelig J et al. High-risk chronic lymphocytic leukemia in the era of pathway inhibitors: integrating molecular and cellular therapies. Blood. 2018;132(9): 892-902.

39. Krämer I, Stilgenbauer S, Dietrich S, et al Allogeneic hematopoietic cell transplantation for high-risk CLL: 10-year follow-up of the GCLLSG CLL3X trial. Blood 2017;130(12):1477-1480.

40. Link CS, Teipel R, Heidenreich F, et al Durable responses to ibrutinib in patients with relapsed CLL after allogeneic stem cell transplantation. Bone Marrow Transplant. 2016;51(6):793-798.

41. Ryan CE, Sahaf B, Logan AC, et al. Ibrutinib efficacy and tolerability in patients with relapsed chronic lymphocytic leukemia following allogeneic HSCT. Blood 2016;128(25):2899-2908.

42. Dreger P, Schetelig J, Andersen N, et al. Managing high-risk CLL during transition to a new treatment era: stem cell transplantation or novel agents? Blood. 2014;124(26) 3841-3849.

43. Hoffmann A, Dietrich S, Hain S, et al Allogeneic transplantation in high-risk chronic lymphocytic leukemia: a single-center, intent-to-treat analysis. Haematologica. 2019;104(7):e304-e306. 